\title{
Nonlinear Vibrations of Viscoelastic Plates of Fractional Derivative Type: An AEM Solution
}

\author{
Nick G. Babouskos ${ }^{1}$ and John T. Katsikadelis*,2 \\ ${ }^{1}$ School of Civil Engineering National Technical University of Athens, Athens, GR-15773, Greece \\ ${ }^{2}$ Office of Theoretical and Applied Mechanics, Academy of Athens \& School of Civil Engineering National Technical \\ University of Athens, Athens, GR-15773, Greece
}

\begin{abstract}
The nonlinear dynamic response of thin plates made of linear viscoelastic material of fractional derivative type is investigated. The resulting governing equations are three coupled nonlinear fractional partial differential equations in terms of displacements. The solution is achieved using the AEM. According to this method the original equations are converted into three uncoupled linear equations, namely a biharmonic (linear thin plate) equation for the transverse deflection and two Poisson's (linear membrane) equations for the inplane deformation under time dependent fictitious loads. The resulting thus initial value problems for the fictitious loads is a system of nonlinear fractional ordinary differential equations, which is solved using the numerical method developed recently by Katsikadelis for multi-term fractional differential equations. Several plates subjected to various loads and boundary conditions are analyzed and the influence of the viscoelastic character of the material is investigated. Without excluding other viscoelastic models, the viscoelastic material employed herein is described by the generalized Voigt model of fractional order derivative. The numerical results demonstrate the efficiency and validate the accuracy of the solution procedure. Emphasis is given to the resonance response of viscoelastic plates under harmonic excitation, where complicated phenomena, similar to those of Duffing equation occur.
\end{abstract}

Keywords: Plates, viscoelasticity, fractional viscoelastic models, nonlinear vibrations, nonlinear resonance, boundary elements, analog equation method, fractional partial differential equations, numerical solution.

\section{INTRODUCTION}

Thin plates of viscoelastic material are extensively used as structural members in many modern engineering applications such as aircrafts, ships and other industrial structures. They are exposed to severe lateral and inplane loads which may produce large amplitude vibrations. In many cases the external load is varying harmonically with time. Resonance may occur when the frequency of the external excitation is close to the lower natural frequencies of the plate. In this case the linear plate theory predicts unbounded oscillations. However, due to the nonlinear character of the problem and the presence of damping, large oscillations remain within certain limits, and jump phenomena on the amplitude of the oscillations may appear as the external monochromatic excitation varies. Such abrupt variations on the plate response are extremely dangerous and have to be avoided in real structures.

Viscoelastic materials, such as polymers, exhibit both elastic and viscous character and have the advantage of light weight and high strength. There have been proposed various linear and nonlinear models for the mechanical behavior of these materials, such as Maxwell, Voigt, Zener and multiparameter models. The constitutive equations can be presented in differential or in hereditary integral form. Recently,

*Address correspondence to this author at the Office of Theoretical and Applied Mechanics, Academy of Athens \& School of Civil Engineering National Technical University of Athens, Athens, GR-15773, Greece; Tel: 210-7721652; Fax: 210-7721001; E-mail: jkats@central.ntua.gr many researchers have shown that differential viscoelastic models with fractional derivatives are in better agreement with the experimental results than the integer derivative models [1-3].

The nonlinear oscillations of single and multi-degree of freedom systems have been examined thoroughly by many authors such as Nayfeh and Mook [4] and Fang and Dowell [5]. The nonlinear dynamic plate problem is investigated in several papers. Chu and Herrman [6] were among the first who studied large amplitude free vibrations and nonlinear resonance of elastic plates using a perturbation technique. Sridhar et al. [7] investigated nonlinear resonances of forced circular plates using the method of multiple scales. Amabili [8] studied nonlinear vibrations of rectangular plates using Rayleigh-Ritz method. He investigated the influence of inplane boundary conditions on the nonlinear resonance and compared his results with experimental ones. Many other researchers studied the large amplitude vibrations of thin elastic rectangular and circular plates using approximate techniques (Galerkin method) [9-11]. The FEM has proven to be a powerful method for the solution of the nonlinear oscillations of complicated structures. Lee and $\mathrm{Ng}$ [12] studied the nonlinear response of isotropic and composite plates using FEM and a modal reduction method in order to reduce the degrees of freedom. Ribeiro and Petyt $[13,14]$ used FEM and the harmonic balance method to study rectangular plates subjected to harmonic excitations. They examined the influence of inplane and transverse boundary conditions on resonance and compared their results with experimental findings. 
The BEM has been also used for the nonlinear dynamic analysis of thin plates [15-17].

The literature on nonlinear response of viscoelastic plates is rather limited. Ioannides and Grootenhius [18] used the FEM to study harmonic excitations of layered plates with a viscoelastic core. Eshmatov et al. $[19,20]$ studied the nonlinear vibrations and dynamic stability of viscoelastic plates with integral constitutive equations using Bubnov-Galerkin method. Rossikhin and Shitikova [21] analyzed the nonlinear vibrations of viscoelastic plates with damping modeled with fractional derivatives. The resulting fractional differential equations are solved using a finite difference approximation for the fractional terms.

In this paper the nonlinear dynamic response of thin viscoelastic plates of fractional derivative model is studied. The plate has an arbitrary shape and is subjected to any type of boundary conditions and loading. The governing equations result by taking the equilibrium of the plate element in a slightly deformed configuration (moderate large deflections). Fractional differential constitutive equations are employed. Without restricting the generality the generalized Voigt model is employed. The resulting equations of motion are three coupled nonlinear partial fractional differential equations in terms of the three displacement components. The nonlinearity is due to nonlinear kinematic relations based on the von Kármán assumption. The solution is achieved using the Analog Equation Method (AEM) [22]. The concept of the analog equation of Katsikadelis is applied to convert the original three coupled nonlinear fractional partial differential equations (PDE's) into three uncoupled linear equations, namely a biharmonic (linear thin plate) equation for the transverse deflection and two Poisson's (linear membrane) equations for the inplane deformation under time dependent fictitious loads that are unknown in the first instance. Subsequent use of the BEM results in the initial value problem for the fictitious sources, which constitutes a system of nonlinear fractional ordinary differential equations including third order nonlinear terms. Its solution is achieved using the numerical method developed recently by Katsikadelis [23] for multi-term linear and nonlinear fractional differential equations. Several plates subjected to various loads and boundary conditions are analyzed and the influence of the viscoelastic character of the material is studied. The worked out examples illustrate the method and demonstrate its efficiency. Emphasis is given in the study of the resonance response of the viscoelastic plate under harmonic excitation, where phenomena similar to those of Duffing's equation occur. In conclusion, the developed method provides a computational tool for a systematic analysis of viscoelastic plates under large displacements permitting thus a better insight in their response.

\section{DERIVATION OF THE GOVERNING EQUATIONS}

Consider a thin plate of uniform thickness $h$ of linear viscoelastic material occupying the two dimensional multiply connected domain $\Omega$ of the $x y$ plane with boundary

$\Gamma=\cup_{i=0}^{K} \Gamma_{i}$ (Fig. 1). The curves $\Gamma_{i}(i=0,1,2, \ldots, K)$ may be piece-wise smooth. The boundary may be simply supported, clamped, free or elastically supported with transverse stiffness $k_{T}(\mathbf{x})$ and rotational stiffness $k_{R}(\mathbf{x}) \mathbf{x}:(x, y) \in \Gamma$.
The plate is subjected to transverse $p_{z}$ and inplane loads $p_{x}, p_{y}$ (body forces).

Without restricting the generality, it is assumed that the material is described by the generalized Voigt model. The three dimensional viscoelastic differential constitutive equations for this model read [24]

$$
\begin{aligned}
& s_{i j}=2 \mu e_{i j}+2 \mu \eta D_{c}^{\alpha} e_{i j} \\
& \sigma_{k k}=3 K \varepsilon_{k k}+3 K \eta D_{c}^{\alpha} \varepsilon_{k k}
\end{aligned}
$$

where $s_{i j}, e_{i j}$ are the deviatoric components and $\sigma_{k k}, \varepsilon_{k k}$ the dilatational components of stress and strain respectively, with $\quad i, j, k=1,2,3 \quad(1 \equiv x, 2 \equiv y, 3 \equiv z)$; $\mu=G=E / 2(1+\nu)$ and $K=E / 3(1-2 \nu)$ are the shear and bulk modulus, respectively, with $E, \nu$ being the engineering constants; $\eta$ is the viscoelastic parameter and $D_{c}^{a}$ the Caputo fractional derivative of order $\alpha$ defined as [25]

$$
D_{c}^{\alpha} u(t)=\left\{\begin{array}{cl}
{\left[\frac{1}{\Gamma(m-\alpha)} \int_{0}^{t} \frac{u^{(m)}(\tau)}{(t-\tau)^{\alpha+1-m}} d \tau\right],} & m-1<\alpha<m \\
\frac{d^{m}}{d t^{m}} u(t) & m=\alpha
\end{array}\right.
$$

The relations between the deviatoric and dilatational components are given as [26]

$s_{i j}=\sigma_{i j}-\frac{1}{3} \sigma_{k k} \delta_{i j} e_{i j}=\varepsilon_{i j}-\frac{1}{3} \varepsilon_{k k} \delta_{i j}$

The assumptions of the Kirchhoff plate theory are stated as

$\sigma_{33}=\varepsilon_{23}=\varepsilon_{13}=0$

Using Eqs (3) and (4) the constitutive equations (1a,b) become

$$
\begin{aligned}
& \sigma_{11}=\frac{\mathrm{E}}{\left(1-\nu^{2}\right)}\left(\varepsilon_{11}+\nu \varepsilon_{22}\right)+\frac{\eta \mathrm{E}}{\left(1-\nu^{2}\right)}\left(D^{a} \varepsilon_{11}+\nu D^{a} \varepsilon_{22}\right) \\
& \sigma_{22}=\frac{\mathrm{E}}{\left(1-\nu^{2}\right)}\left(\nu \varepsilon_{11}+\varepsilon_{22}\right)+\frac{\eta \mathrm{E}}{\left(1-\nu^{2}\right)}\left(\nu D^{a} \varepsilon_{11}+D^{a} \varepsilon_{22}\right) \\
& \sigma_{12}=\frac{E}{1+\nu} \varepsilon_{12}+\eta \frac{E}{1+\nu} D^{a} \varepsilon_{12}
\end{aligned}
$$

Moderate large deflections are considered where the von Kármán assumption for the kinematic relation is adopted. That is

$$
\begin{aligned}
& \varepsilon_{11}=u,_{x}+\frac{1}{2} w,_{x}^{2} \varepsilon_{22}=v,_{y}+\frac{1}{2} w,_{y}^{2} \\
& \varepsilon_{12}=\frac{1}{2}\left(u,_{y}+v,_{x}+w,_{x} w,_{y}\right)
\end{aligned}
$$

where $u=u(x, y, t), v=v(x, y, t)$ are the membrane and $w=w(x, y, t)$ the transverse displacements. 
On the basis of the Eqs (5) and (6) the stress resultants are written as

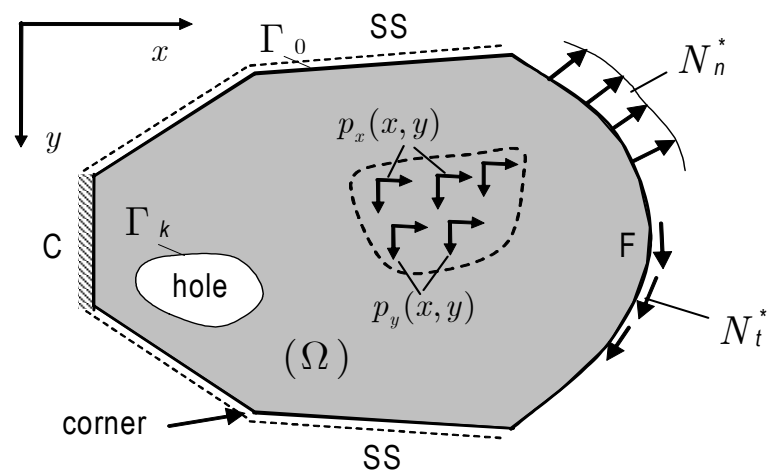

Fig. (1). Plate geometry and supports $(\mathrm{C}=$ clamped, $\mathrm{SS}=$ simply supported, $\mathrm{F}=$ free)

$$
\begin{aligned}
& N_{x}=C\left[u,_{x}+\nu v,_{y}+\frac{1}{2}\left(w,_{x}^{2}+\nu w,_{y}^{2}\right)\right]+ \\
& \eta C\left[D_{c}^{\alpha} u,_{x}+\nu D_{c}^{\alpha} v_{,_{y}}+\frac{1}{2} D_{c}^{\alpha}\left(w_{,_{x}}^{2}+\nu w,_{y}^{2}\right)\right] \\
& N_{y}=C\left[\nu u,_{x}+v,_{y}+\frac{1}{2}\left(\nu w_{,_{x}}^{2}+w,_{y}^{2}\right)\right]+ \\
& \eta C\left[\nu D_{c}^{\alpha} u,_{x}+D_{c}^{\alpha} v,_{y}+\frac{1}{2} D_{c}^{\alpha}\left(\nu w,_{x}^{2}+w,_{y}^{2}\right)\right] \\
& N_{x y}=C \frac{1-\nu}{2}\left(u,_{y}+v,_{x}+w,_{x} w,_{y}\right)+ \\
& \eta C \frac{1-\nu}{2}\left[D_{c}^{\alpha} u,_{y}+D_{c}^{\alpha} v,_{x}+\frac{1}{2} D_{c}^{\alpha}\left(w,_{x} w,_{y}\right)\right] \\
& M_{x}=-D\left(w,_{x x}+\nu w,_{y y}\right)-\eta D\left(D_{c}^{\alpha} w,_{x x}+\nu D_{c}^{\alpha} w_{y y}\right) \\
& M_{y}=-D\left(\nu w,_{x x}+w,_{y y}\right)-\eta D\left(\nu D_{c}^{\alpha} w,_{x x}+D_{c}^{\alpha} w,_{y y}\right) \\
& M_{x y}=-D(1-\nu) w,_{x y}-\eta D(1-\nu) D_{c}^{\alpha} w,_{x y}
\end{aligned}
$$

where $C=E h /\left(1-\nu^{2}\right)$ is the membrane stiffness and $D=E h^{3} / 12\left(1-\nu^{2}\right)$ is the flexural stiffness of the plate. The governing equations result by taking the equilibrium of the plate element in a slightly deformed configuration. This yields

$$
\begin{aligned}
& \frac{\partial^{2} M_{x x}}{\partial x^{2}}+2 \frac{\partial^{2} M_{x y}}{\partial x \partial y}+\frac{\partial^{2} M_{y y}}{\partial x^{2}}+\frac{\partial}{\partial x}\left(N_{x} w,_{x}+N_{x y} w,_{y}\right) \\
&+\frac{\partial}{\partial y}\left(N_{x y} w,_{x}+N_{y} w,_{y}\right)+p_{z}-\rho h \ddot{w}=0 \\
& \frac{\partial N_{x}}{\partial x}+\frac{\partial N_{x y}}{\partial y}+p_{x}-\rho h \ddot{u}=0
\end{aligned}
$$

$$
\frac{\partial N_{x y}}{\partial x}+\frac{\partial N_{y}}{\partial y}+p_{y}-\rho h \ddot{v}=0
$$

Using Eqs (7), (8) and (9) we obtain the plate equations in terms of displacements in $\Omega$ as

\section{i. For the Transverse Deflection}

$$
\begin{aligned}
D \nabla^{4} w+\eta D D_{c}^{\alpha} \nabla^{4} w-N_{x} w,_{x x}-2 N_{x y} w,_{x y}-N_{y} w,_{y y} \\
\quad\left(p_{x}-\rho h \ddot{u}\right) w,_{x}+\left(p_{y}-\rho h \ddot{v}\right) w,_{y}+\rho h \ddot{w}=p_{z}
\end{aligned}
$$

\section{ii. For the Inplane Deformation}

$$
\begin{aligned}
& \nabla^{2} u+\frac{1+\nu}{1-\nu}\left(u,_{x}+v,_{y}\right),_{x}+w,,_{x}\left(\frac{2}{1-\nu} w,_{x x}+w,_{y y}\right)+\frac{1+\nu}{1-\nu} w,_{x y} w,_{y} \\
& +\eta D_{c}^{\alpha}\left[\begin{array}{l}
\nabla^{2} u+\frac{1+\nu}{1-\nu}\left(u_{x_{x}}+v_{,_{y}}\right),_{x}+ \\
w_{x}\left(\frac{2}{1-\nu} w_{x x}+w_{y y}\right)+\frac{1+\nu}{1-\nu} w,_{x y} w,_{y}
\end{array}\right] \\
& +\frac{p_{x}}{G h}-\frac{\rho}{G} \ddot{u}=0 \\
& \nabla^{2} v+\frac{1+\nu}{1-\nu}\left(u,_{x}+v,_{y}\right)_{,_{y}}+w,_{y}\left(\frac{2}{1-\nu} w_{y y}+w,_{x x}\right)+ \\
& \frac{1+\nu}{1-\nu} w,_{x y} w,_{x}+\eta D_{c}^{\alpha}\left[\begin{array}{l}
\nabla^{2} v+\frac{1+\nu}{1-\nu}\left(u,_{x}+v,_{y}\right),_{y}+ \\
w,_{y}\left(\frac{2}{1-\nu} w,_{y y}+w,_{x x}\right)+\frac{1+\nu}{1-\nu} w,_{x y} w,_{x}
\end{array}\right] \\
& +\frac{p_{y}}{G h}-\frac{\rho}{G} \ddot{v}=0
\end{aligned}
$$

The associated boundary conditions result as [27]

$V_{n}+\eta D_{c}^{\alpha} V_{n}+\bar{N}_{n} w,_{n}+\bar{N}_{t} w_{{ }_{t}}+k_{T} w=\bar{V}_{n}$ or $w=\bar{w}$ on $\Gamma$

$M_{n}+\eta D_{c}^{\alpha} M_{n}-k_{R} w,_{n}=\bar{M}_{n}$ or $w,_{n}=\bar{w}_{,_{n}}$ on $\Gamma$

$k_{T}^{(k)} w^{(k)}-\llbracket M_{n t} \rrbracket_{k}-\eta D_{c}^{\alpha}\left(\llbracket M_{n t} \rrbracket_{k}\right)=\bar{R}_{k} \quad$ or $\quad w^{(k)}=\bar{w}_{k} \quad$ at corner point $\mathrm{k}$

$N_{n}=\bar{N}_{n}$ or $u_{n}=\bar{u}_{n}$ on $\Gamma$

$N_{t}=\bar{N}_{t}$ or $u_{t}=\bar{u}_{t}$ on $\Gamma$

where the overbar designates a prescribed quantity. Moreover, the viscoelastic plate is subjected to the initial conditions

$$
\begin{aligned}
& w(\mathbf{x}, 0)=g_{1}(\mathbf{x}), \dot{w}(\mathbf{x}, 0)=q_{1}(\mathbf{x}), \text { in } \Omega \\
& u(\mathbf{x}, 0)=g_{2}(\mathbf{x}), \dot{u}(\mathbf{x}, 0)=q_{2}(\mathbf{x}), \text { in } \Omega \\
& v(\mathbf{x}, 0)=g_{3}(\mathbf{x}), \dot{v}(\mathbf{x}, 0)=q_{3}(\mathbf{x}) \text { in } \Omega
\end{aligned}
$$

In Eqs (11), $V_{n}=V w$ is the equivalent shear force, $M_{n}=M w$ is the normal bending moment; $M_{n t}=T w$ is the twisting moment along the boundary and $\llbracket M_{n t} \rrbracket_{k}$ is its discontinuity jump at corner $k$. The operators producing these quantities are given as [28] 


$$
\begin{aligned}
& V=-D\left[\frac{\partial}{\partial n} \nabla^{2}+(1-\nu) \frac{\partial}{\partial s}\left(\frac{\partial^{2}}{\partial s \partial n}-\kappa \frac{\partial}{\partial s}\right)\right] \\
& M=-D\left[\nabla^{2}-(1-\nu)\left(\frac{\partial^{2}}{\partial s^{2}}+\kappa \frac{\partial}{\partial n}\right)\right] \\
& T=D(1-\nu)\left(\frac{\partial^{2}}{\partial s \partial n}-\kappa \frac{\partial}{\partial s}\right)
\end{aligned}
$$

where $\kappa=\kappa(s)$ is the curvature of the boundary and $n, s$ the intrinsic boundary coordinates, i.e. the coordinates along the normal and tangential directions at a boundary point.

\section{THE AEM SOLUTION}

\subsection{The Plate Problem}

The initial boundary value problem (10a), (11a,b,c) and $(12 a, b)$ for the response of the plate is solved using the AEM. Noting that Eq. (10a) is of fourth order with respect to $w$, the analog equation for the transverse displacement is

$\nabla^{4} w=b(\mathbf{x}, t), \quad \mathbf{x}=\{x, y\} \in \Omega$

where $b(\mathbf{x}, t)$ represents the time dependent fictitious load, unknown in the first instance. Eq. (16) is a quasi-static equation, that is the time appears as a parameter, and it can be solved with the boundary conditions $(11 \mathrm{a}, \mathrm{b}, \mathrm{c})$ at any instant $t$ using the BEM. Thus, the solution at a point $\mathbf{x} \in \Omega$ is obtained in integral form as

$$
\begin{array}{r}
w(\mathbf{x}, t)=\int_{\Omega} w^{*} b d \Omega+\int_{\Gamma}\left(w^{*} V_{n}+w{ }_{n} M_{n}^{*}-w^{*}{ }_{n} M_{n}-w V_{n}^{*}\right) d s \\
-\sum_{k}\left(w^{*} \llbracket M_{n t} \rrbracket-w \llbracket M_{n t}^{*} \rrbracket\right)_{k}
\end{array}
$$

which for $\mathbf{x} \in \Gamma$ yields the following two boundary integral equations for points where the boundary is smooth

$$
\begin{array}{r}
\frac{1}{2} w(\mathbf{x}, t)=\int_{\Omega} w^{*} b d \Omega+ \\
\int_{\Gamma}\left(w^{*} V_{n}+w,_{n} M_{n}^{*}-w^{*},_{n} M_{n}-w V_{n}^{*}\right) d s \\
-\sum_{k}\left(w^{*} \llbracket M_{n t} \rrbracket-w \llbracket M_{n t}^{*} \rrbracket\right)_{k} \\
\frac{1}{2} w_{\nu}(\mathbf{x}, t)=\int_{\Omega} w_{1}^{*} b d \Omega+ \\
\int_{\Gamma}\left(w_{1}^{*} V_{n}+w,_{n} M_{n 1}^{*}-w_{1}^{*},_{n} M_{n}-w V_{n 1}^{*}\right) d s \\
-\sum_{k}\left(w_{1}^{*} \llbracket M_{n t} \rrbracket-w \llbracket M_{n t 1}^{*} \rrbracket\right)_{k}
\end{array}
$$

where $\quad V_{n}^{*}=V w^{*}, \quad M_{n}^{*}=M w^{*}, \quad M_{n t}^{*}=T w^{*} \quad$ and $V_{1 n}^{*}=V w_{1}^{*}, \quad M_{n 1}^{*}=M w_{1}^{*}, \quad M_{n t 1}^{*}=T w_{1}^{*} \quad$ in $\quad$ which $w^{*}=w^{*}(\mathbf{x}, \mathbf{y}), \mathbf{x}, \mathbf{y} \in \Gamma$, is the fundamental solution and $w_{1}^{*}$ its normal derivative at point $\mathbf{x} \in \Gamma$ in the direction $\boldsymbol{\nu}$, i.e.

$$
w^{*}=\frac{1}{8 \pi} r^{2} \ln r \quad w_{1}^{*}=\left(\frac{1}{8 \pi} r^{2} \ln r\right), \nu=\frac{1}{8 \pi} r r_{,}(2 \ln r+1)
$$

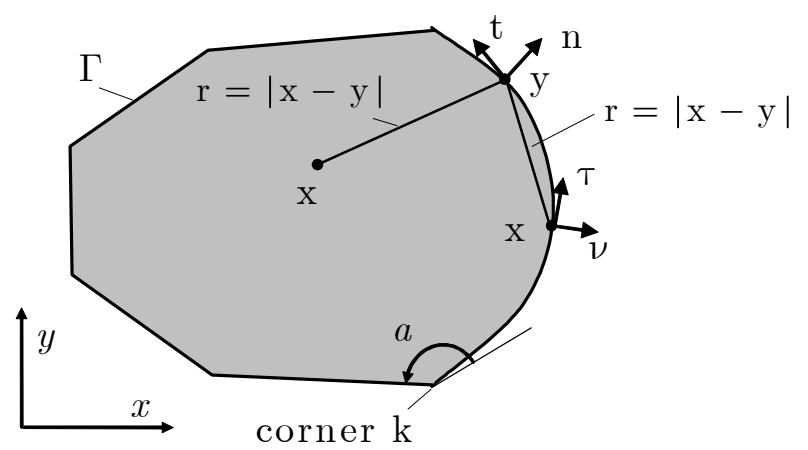

Fig. (2). BEM notation.

$\boldsymbol{\nu}$ is the unit normal vector to the boundary at point $\mathrm{x}$, whereas $\mathbf{n}$ is the unit normal vector to the boundary at the integration point $\mathbf{y}$ and $r=|\mathbf{x}-\mathbf{y}|$ (see Fig. 2). Eqs (18) and (19) can be used to establish the not specified boundary quantities. They are solved numerically using the BEM. The boundary integrals are approximated using $N$ constant boundary elements, whereas the domain integrals are approximated using $M$ linear triangular elements. The domain discretization is performed automatically using the Delaunay triangulation [29]. Since the fictitious source is not defined on the boundary, the nodal points of the triangles adjacent to the boundary are placed on their sides (Fig. 3).

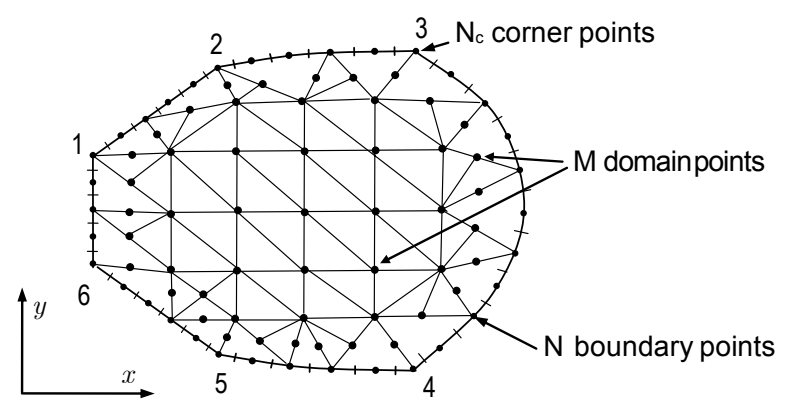

Fig. (3). Boundary and domain discretization.

Thus, after discretization and application of the boundary integral Eqs (18) and (19) at the $N$ boundary nodal points and Eq. (18) at the $N_{c}$ corner points we obtain

$\mathbf{H}\left\{\begin{array}{c}\mathbf{w} \\ \mathbf{w}_{c} \\ \mathbf{w}_{,_{n}}\end{array}\right\}=\mathbf{G}\left\{\begin{array}{l}\mathbf{v} \\ \mathbf{R} \\ \mathbf{M}\end{array}\right\}+\mathbf{A b}$

where

H,G : are $N \times N$ known coefficient matrices originating from the integration of the kernel functions on the boundary elements.

A :is an $N \times M$ coefficient matrix originating from the integration of the kernel function on the domain elements. 
$\mathbf{w}, \mathbf{w}_{c}, \mathbf{w}_{n}$ : are the vectors of the $N$ boundary nodal displacements, $N_{c}$ corner displacements and $N$ boundary nodal normal slopes, respectively.

$\mathbf{V}, \mathbf{R}, \mathbf{M}$ : are the vectors of the $N$ nodal values of effective shear force, $N_{c}$ concentrated corner forces and $N$ nodal values of the normal bending moment.

$\mathbf{b}$ : is the vector of the $M$ nodal values of the fictitious source inside $\Omega$.

Eq. (21) constitutes a system of $2 N+N_{c}$ equations for $4 N+2 N_{c}+M$ unknowns. Additional $2 N+N_{c}$ equations are obtained from the boundary conditions. Thus, the BCs (11a)-(11c), when applied at the $N$ boundary nodal points and the $N_{c}$ corner points yield the set of equations

$$
\begin{aligned}
& \boldsymbol{\alpha}_{1} \mathbf{w}+\boldsymbol{\alpha}_{2} \mathbf{w},_{n}+\boldsymbol{\alpha}_{3} \mathbf{V}=\boldsymbol{\alpha}_{4} \quad \boldsymbol{\beta}_{1} \mathbf{w}_{,_{n}}+\boldsymbol{\beta}_{2} \mathbf{M}=\boldsymbol{\beta}_{3} \\
& \mathbf{c}_{1} \mathbf{w}_{c}+\mathbf{c}_{2} \mathbf{R}=\mathbf{c}_{3}
\end{aligned}
$$

where $\boldsymbol{\alpha}_{1}, \boldsymbol{\alpha}_{2}, \boldsymbol{\alpha}_{3}, \boldsymbol{\alpha}_{4}, \boldsymbol{\beta}_{1}, \boldsymbol{\beta}_{2}, \boldsymbol{\beta}_{3}, \mathbf{c}_{1}, \mathbf{c}_{2}, \mathbf{c}_{3}$ are known coefficient matrices. The time dependent terms of the boundary conditions are neglected in this investigation and their influence will be the subject of further research. Note that Eq. (22a) has resulted after approximating the derivative $w_{t}$ in Eq. (11a) with a finite difference scheme.

Equations (21) and (22) can be combined and solved for the boundary quantities $\mathbf{w}, \mathbf{w}_{c}, \mathbf{w},_{n}, \mathbf{V}, \mathbf{R}, \mathbf{M}$ in terms of the fictitious load $\mathbf{b}$. Subsequently, these expressions are used to eliminate the boundary quantities from the discretised counterpart of Eq. (17). Thus we obtain the following representation for the deflection

$$
w(\mathbf{x}, t)=\sum_{k=1}^{M} b_{k}(t) W_{k}(\mathbf{x})+W_{0}(\mathbf{x}) \quad \mathbf{x} \in \Omega
$$

The derivatives of $w(\mathbf{x})$ at points $\mathbf{x}$ inside $\Omega$ are obtained by direct differentiation of Eq. (17). Thus, we obtain after elimination of the boundary quantities

$$
\begin{aligned}
& w,_{p q r}(\mathbf{x}, t)=\sum_{k=1}^{M} b_{k}(t) W_{k},_{p q r}(\mathbf{x})+W_{0},_{p q r}(\mathbf{x}), \\
& p, q, r=0, x, y \quad \mathbf{x} \in \Omega
\end{aligned}
$$

where $W_{k},_{p q r}(\mathbf{x})$ are known functions. The term $W_{0}$ result from the nonhomogeneous boundary conditions. Note that the above notation implies $w_{, 000}=w, w,_{0 y 0}=w,_{y}$, etc.

\subsection{The Plane Stress Problem}

Noting that Eqs (10b) and (10c) are of the second order with respect to the displacements $u$ and $v$, their analog equations are obtained using the Laplace operator. This yields

$$
\nabla^{2} u=b_{1}(\mathbf{x}, t) \quad \nabla^{2} v=b_{2}(\mathbf{x}, t)
$$

The integral representation of the solution of Eq. (25a) is

$$
\varepsilon u(\mathbf{x})=\int_{\Omega} v^{*} b_{1} d \Omega-\int_{\Gamma}\left(v^{*} q-q^{*} u\right) d s \quad \mathbf{x} \in \Omega \cup \Gamma
$$

in which $q=u,_{n} ; v^{*}=\ell n r / 2 \pi$ is the fundamental solution to Eq. (25a) and $q^{*}=v,_{n}^{*}$ its derivative normal to the boundary; $r=|\mathbf{y}-\mathbf{x}| \mathbf{x} \in \Omega \cup \Gamma$ and $\mathbf{y} \in \Gamma ; \varepsilon$ is the free term coefficient ( $\varepsilon=1$ if $\mathbf{x} \in \Omega, \varepsilon=1 / 2$ if $\mathbf{x} \in \Gamma$ and $\varepsilon=0$ if $\mathbf{x} \notin \Omega \cup \Gamma$ ). Using the BEM with constant boundary elements and linear triangular domain elements and following the same procedure applied for the plate equation, we obtain the following representation for the inplane displacement $u$ and its derivatives

$$
\begin{aligned}
& u,_{p q}(\mathbf{x}, t)=\sum_{k=1}^{M} b_{k}^{(1)}(t) U_{k}^{(1)},_{p q}(\mathbf{x})+\sum_{k=1}^{M} b_{k}^{(2)}(t) U_{k}^{(2)},_{p q}(\mathbf{x})+U_{0},_{p q}(\mathbf{x}) \\
& , p, q=0, x, y \quad \mathbf{x} \in \Omega
\end{aligned}
$$

Similarly, we obtain for the displacement $v$

$$
\begin{aligned}
& v,_{p q}(\mathbf{x}, t)=\sum_{k=1}^{M} b_{k}^{(1)}(t) V_{k}^{(1)},_{p q}(\mathbf{x})+\sum_{k=1}^{M} b_{k}^{(2)}(t) V_{k}^{(2)},_{p q}(\mathbf{x})+V_{0},_{p q}(\mathbf{x}) \\
& , p, q=0, x, y \mathbf{x} \in \Omega
\end{aligned}
$$

where $U_{k}^{(1)}(\mathbf{x}), U_{k}^{(2)}(\mathbf{x}), V_{k}^{(1)}(\mathbf{x}), V_{k}^{(2)}(\mathbf{x}), U_{0}(\mathbf{x}), V_{0}(\mathbf{x})$ are known functions. The terms $U_{0}, V_{0}$ result from the nonhomogeneous boundary conditions.

\subsection{The final Step of the AEM}

Eqs (24), (27) and (28) give the displacements $w(x, t), u(x, t), v(x, t)$ and their derivatives provided that the three fictitious sources $\mathbf{b}(t), \mathbf{b}^{(1)}(t), \mathbf{b}^{(2)}(t)$ are first established. This is achieved by working as following.

Collocating the fractional PDEs (10) at the $M$ internal nodal points and substituting the expressions for the transverse deflection, Eqs (24), and the membrane displacements, Eqs (27) and (28), we obtain the following system of $3 M$ nonlinear fractional ordinary differential equations for $b_{k}(t), b_{k}^{(1)}(t), b_{k}^{(2)}(t),(k=1, . ., M)$

$\mathbf{F}\left(\mathbf{b}, \mathbf{b}^{(1)}, \mathbf{b}^{(2)}, D_{c}^{\alpha} \mathbf{b}, D_{c}^{\alpha} \mathbf{b}^{(1)}, D_{c}^{\alpha} \mathbf{b}^{(2)}, \ddot{\mathbf{b}}, \ddot{\mathbf{b}}^{(1)}, \ddot{\mathbf{b}}^{(2)}\right)=\mathbf{P}$

where $\mathbf{P}=\left\{\begin{array}{lll}\mathbf{p}_{z} & \mathbf{p}_{x} & \mathbf{p}_{y}\end{array}\right\}^{T}$ is the vector of the nodal values of the external loads. The initial conditions Eqs (12)-(14) for $b_{k}(t), b_{k}^{(1)}(t), b_{k}^{(2)}(t)$ become

$$
\begin{aligned}
& \mathbf{b}(0)=\mathbf{W}^{-1} \mathbf{g}_{1}, \mathbf{b}^{(1)}(0)=\mathbf{S}_{1}^{-1} \mathbf{g}_{2}, \mathbf{b}^{(2)}(0)=\mathbf{S}_{2}^{-1} \mathbf{g}_{3} \\
& \dot{\mathbf{b}}(0)=\mathbf{W}^{-1} \mathbf{q}_{1}, \dot{\mathbf{b}}^{(1)}(0)=\mathbf{S}_{1}^{-1} \mathbf{q}_{2}, \dot{\mathbf{b}}^{(2)}(0)=\mathbf{S}_{2}^{-1} \mathbf{q}_{3}
\end{aligned}
$$

where $\mathbf{W}, \mathbf{S}_{1}, \mathbf{S}_{2}$ are $N \times N$ known matrices.

Equations (29) constitute a system of $3 M$ three-term nonlinear FDEs, which are solved using the time step nu- 
merical method for multi-term FDEs developed recently by Katsikadelis [23]. This solution method is concisely presented in the Appendix. The use however, of all the degrees of freedom may be computationally costly and in some cases inefficient due to the relatively large number of coefficients $b_{k}(t), b_{k}^{(1)}(t), b_{k}^{(2)}(t)$. To overcome this difficulty in this investigation, the number of degrees of freedom is reduced using the Ritz transformation, namely

$\left\{\begin{array}{l}\mathbf{b} \\ \mathbf{b}^{(1)} \\ \mathbf{b}^{(2)}\end{array}\right\}=\Psi \mathbf{z}$

where $z_{k}(t),(k=1, . ., L<3 M)$ are new time dependent parameters and $\Psi$ is the $3 M \times L$ transformation matrix. In this investigation the eigenmodes of the linear problem are selected as Ritz vectors [27]. Using Eq. (32), Eqs (29), (30) and (31) are transformed into the following reduced initial value problem of nonlinear FDEs

$\overline{\mathbf{F}}\left(\mathbf{z}, D_{c}^{\alpha} \mathbf{z}, \ddot{\mathbf{z}}\right)=\overline{\mathbf{P}}$

$\mathbf{z}(0)=\left(\Psi^{T} \Psi\right)^{-1} \Psi^{T} \overline{\mathbf{b}}(0), \dot{\mathbf{z}}(0)=\left(\Psi^{T} \Psi\right)^{-1} \Psi^{T} \dot{\overline{\mathbf{b}}}(0)$

where

$\overline{\mathbf{F}}\left(\mathbf{z}, D_{c}^{\alpha} \mathbf{z}, \ddot{\mathbf{z}}\right)=\Psi^{T} \mathbf{F}\left(\mathbf{b}, \mathbf{b}^{(1)}, \mathbf{b}^{(2)}, D_{c}^{\alpha} \mathbf{b}, D_{c}^{\alpha} \mathbf{b}^{(1)}\right.$,

$\left.D_{c}^{\alpha} \mathbf{b}^{(2)}, \ddot{\mathbf{b}}, \ddot{\mathbf{b}}^{(1)}, \ddot{\mathbf{b}}^{(2)}\right)$

$\overline{\mathbf{P}}=\Psi^{T} \mathbf{P}$

$\overline{\mathbf{b}}(0)=\left\{\begin{array}{lll}\mathbf{b}(0) & \mathbf{b}^{(1)}(0) & \mathbf{b}^{(2)}(0)\end{array}\right\}^{T}$,

$\dot{\overline{\mathbf{b}}}(0)=\left\{\begin{array}{lll}\dot{\mathbf{b}}(0) & \dot{\mathbf{b}}^{(1)}(0) \quad \dot{\mathbf{b}}^{(2)}(0)\end{array}\right\}^{T}$

\section{NUMERICAL EXAMPLES}

On the basis of the previously described procedure a FORTRAN code has been written for solving the nonlinear vibrations of viscoelastic plates. The efficiency and accuracy of the method is demonstrated by the following examples.

Example 1. Forced vibrations of a square viscoelastic plate.

The nonlinear vibrations of the square plate with side length $a=4 \mathrm{~m}(0 \leq x, y \leq a)$ and thickness $h=0.1 \mathrm{~m}$ are investigated. All edges are simply supported $(w=M w=0)$ and immovable in the plane of the plate $(u=v=0)$. The results were obtained with $N=204$ boundary elements and $M=137$ internal nodal points resulting from 216 triangular cells (Fig. 4).

First the response of the elastic plate is studied for a uniform transverse load (i) $p_{z}=100 H(t)$ and (ii) $p_{z}=100 \sin (100 t)$. The parameters of the elastic material are $E=21 \times 10^{6} \mathrm{~N} / \mathrm{m}^{2}, \rho=10^{4} \mathrm{~kg} / \mathrm{m}^{3} \quad \nu=0.3$. Figs. (5 and 6) present the time history of the transverse deflection

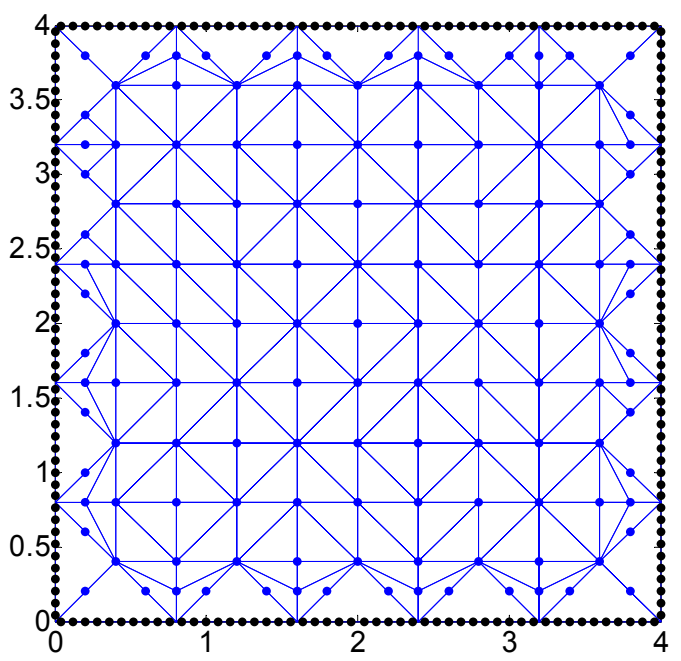

Fig. (4). Boundary and domain discretization ( $N=204$, $M=137$ ).
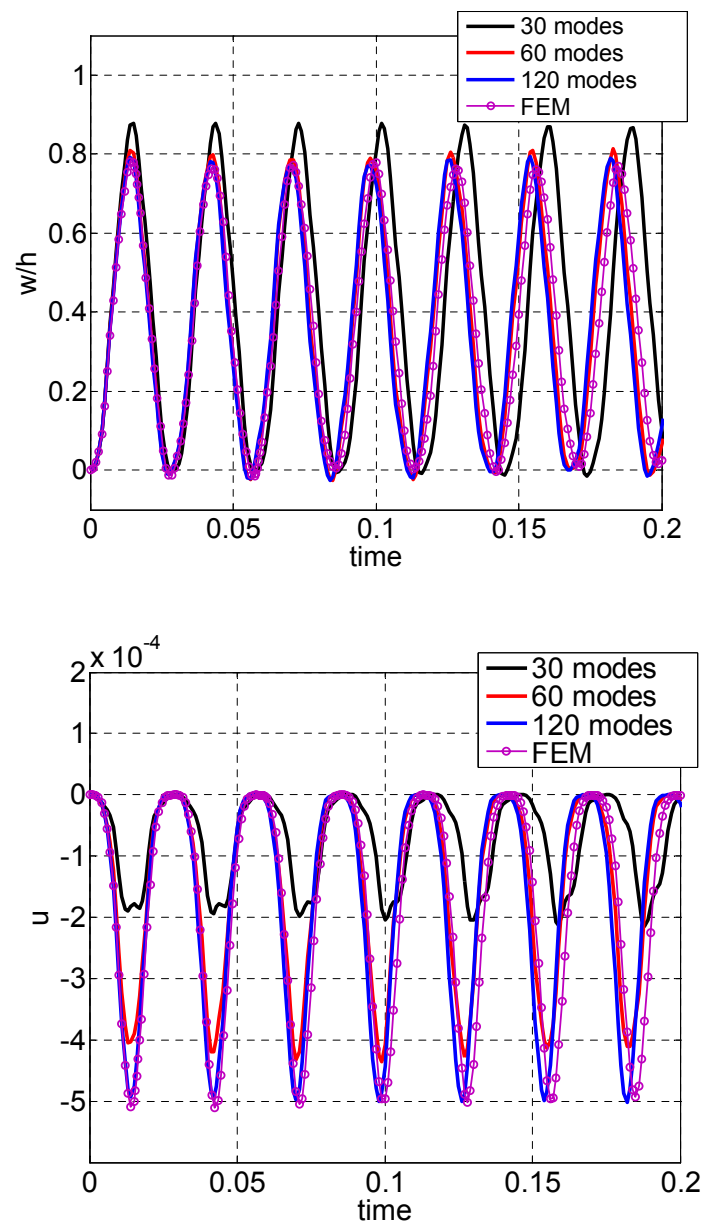

Fig. (5). Elastic plate $(\eta=0)$. Time history of the deflection $w(t)$ at the center of the plate (a) and the inplane displacement $u(t)$ at point $(1,2)(\mathbf{b})$ for elastic material; $p_{z}=100 H(t)$

$w(t)$ at the center of the plate and the inplane displacement $u(t)$ at point $(1,2)$ for load cases (i) and (ii), respectively. 

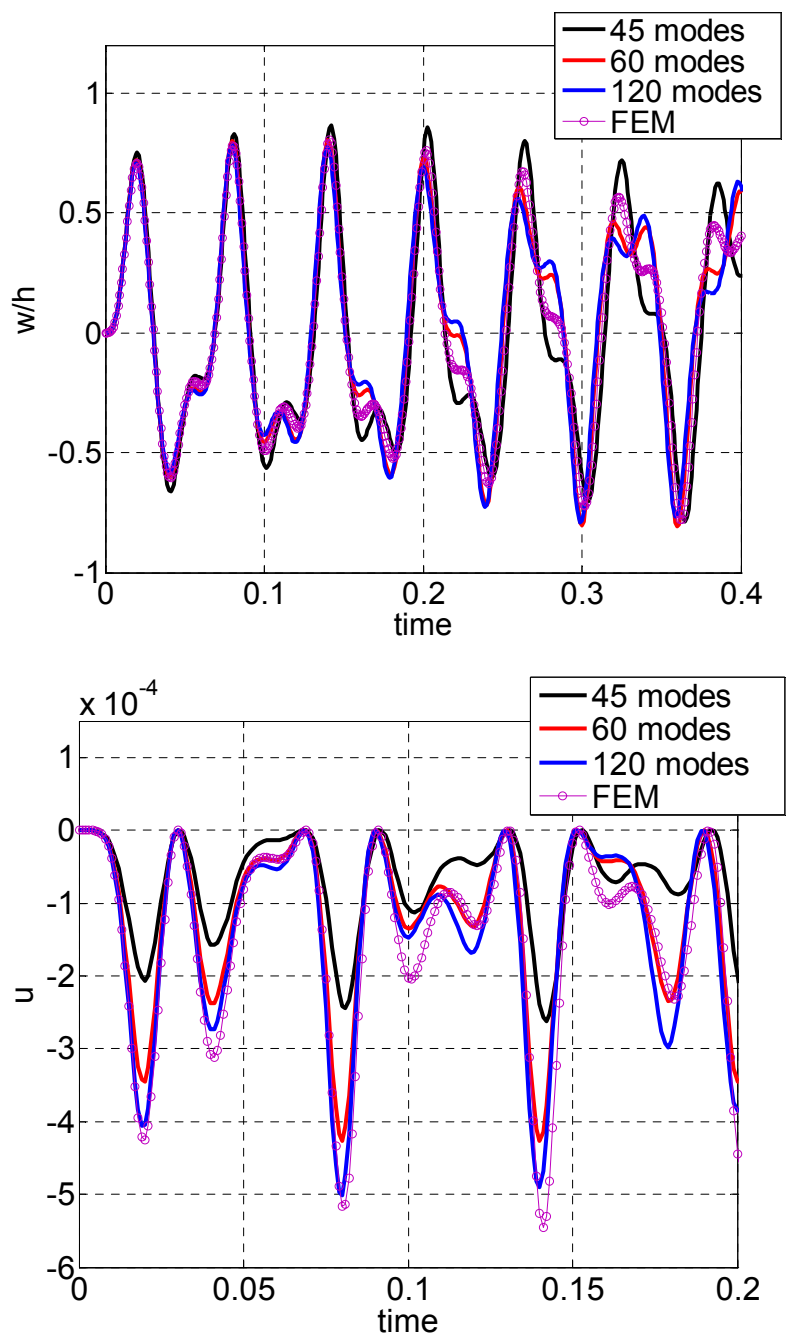

Fig. (6). Elastic plate $(\eta=0)$. Time history of the deflection $w(t)$ at the center of the plate (a) and the inplane displacement $u(t)$ at point $(1,2)(\mathbf{b})$ for elastic material; $p_{z}=100 \sin (100 t)$.

The analysis has been performed using various numbers of linear modes for reduction. The results obtained with 120 modes (total number of modes $3 \times 137$ ) are in good agreement with those obtained from a FEM solution using 1600 quadrilateral elements. Apparently, acceptable results are obtained using only 60 modes for the reduction.

Next, the response of the viscoelastic plate is studied with a viscoelastic parameter $\eta=0.001$ under the uniform loads (i) $p_{z}=100 H(t)$ and (ii) $p_{z}=100 \sin (50 t)$. The results were obtained using 60 modes for reduction of the degrees of freedom. Fig. (7) presents the time history of the deflection $w(t)$ at the center of the plate and the membrane force $N_{x}(t)$ at point $(1,2)$ for load case (i), while Fig. (8) presents the time histories of the same quantities for load case (ii). The analysis has been performed for various values of the order of the fractional derivative model. Since there are no available results in the literature or other numerical solution methods for the problem at hand, a comparison of the results was not possible. The accuracy of the solution procedure has been attested by solving the problem for a very small value of the order $\alpha$ of the fractional derivative and comparing it with the elastic solution with $E^{*}=(1+\eta) E$. Apparently, from Eq. (2) we deduce that $\lim _{\alpha \rightarrow 0} D_{c}^{\alpha} u(t)=u(t)$, therefore on the base of Eqs (1) the material becomes elastic with a substitute elastic modulus $E^{*}=(1+\eta) E$. This comparison is shown in Fig. (9).
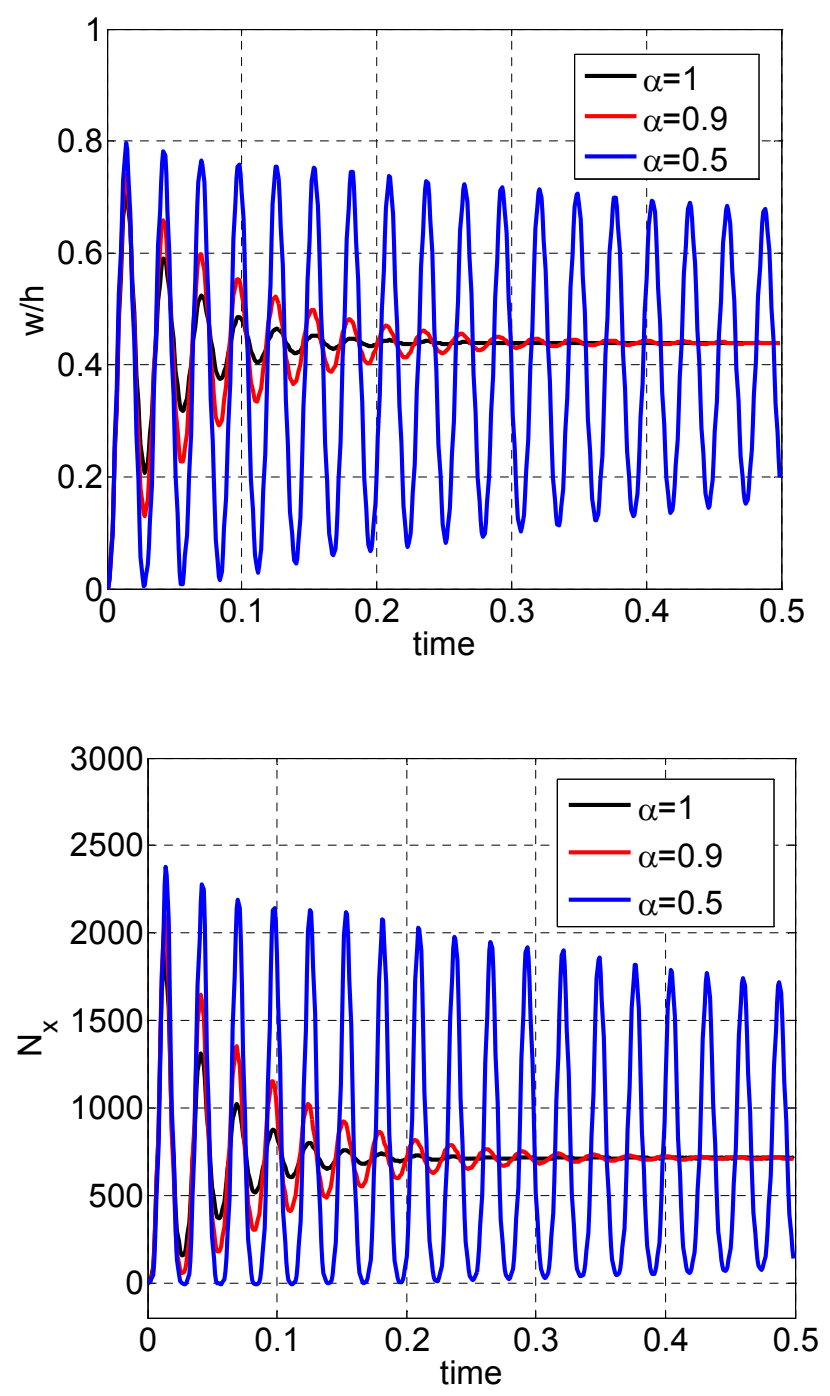

Fig. (7). Viscoelastic plate $(\eta=0.001)$. Time history of the deflection $w(t)$ at the center of the plate (a) and membrane force $N_{x}(t)$ at point $(1,2)$ of plate $(\mathbf{b})$ for various orders of the fractional derivative; $p_{z}=100 H(t)$.

Example 2. Resonance of a viscoelastic square plate

The plate of Example 1 is subjected to a uniform transverse load $p_{z}=\cos (\Omega t)$. All edges are simply supported. Two cases of the inplane boundary conditions were considered. In case (i) the edges are movable in the plane of the 

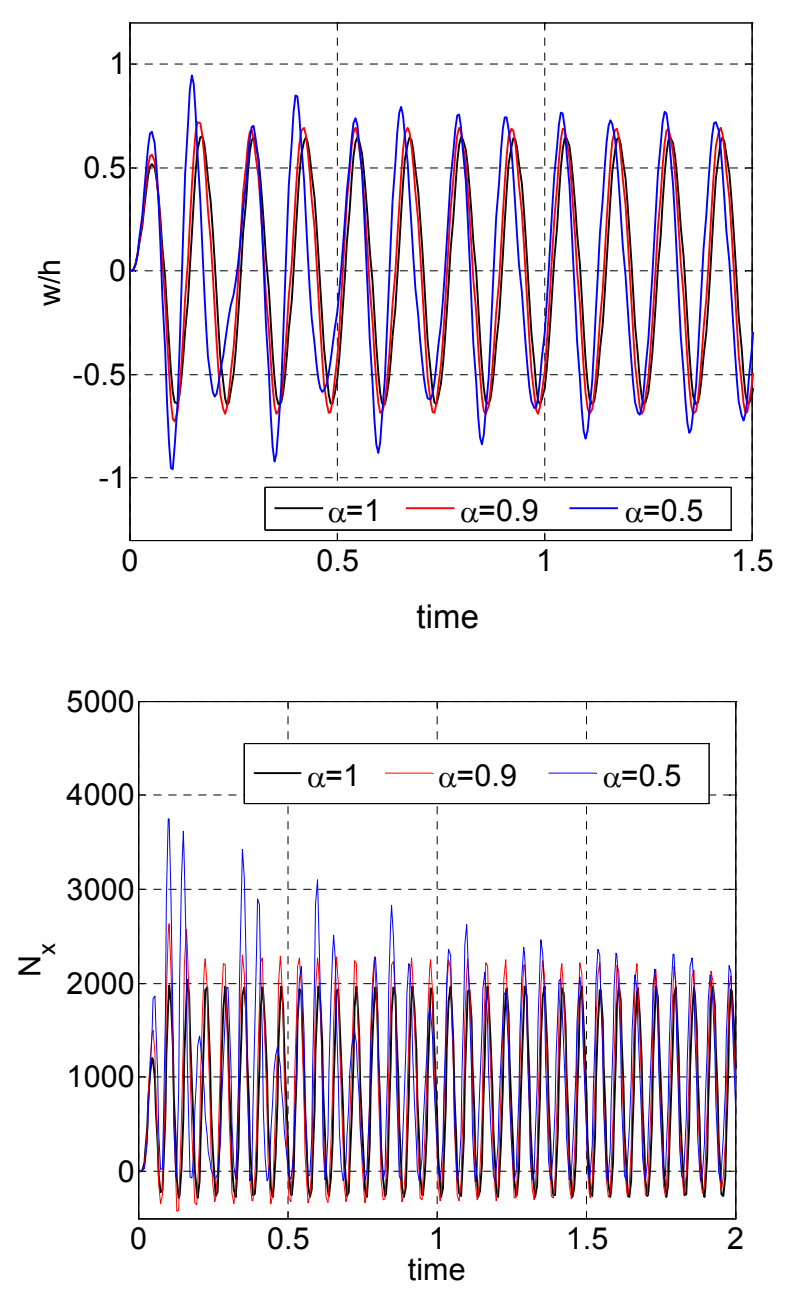

Fig. (8). Viscoelastic plate $(\eta=0.001)$. Time history of the deflection $w(t)$ at the center of the plate (a) and membrane force $N_{x}(t)$ at point $(1,2)$ of the plate $(\mathbf{b})$ for various orders of the fractional derivative; $p_{z}=100 \sin (50 t)$.

plate ( $N_{x}=N_{y}=0$ ), while in case (ii) the edges are restraint $(u=v=0)$. It should be noted that the boundary conditions in case (i) are of Neumann type and the solution is not possible unless the inplane rigid body motion is restrained [30]. This is possible by exploiting the known displacement at the boundary points at axes of symmetry. The results were obtained using 40 linear modes for reduction.

Integrating the equations of motion over a long time is the simplest method to study periodic solutions of nonlinear systems. The system can converge to an either stable solution (attractor) by starting from specific initial conditions. Even though this method is very general, it is difficult to obtain unstable solutions. More accurate methods have been introduced such as the harmonic balance method, the Poincare map and arc-length methods for periodic solutions [31].

In this investigation we study the nonlinear response of the plate over a long time beginning from specific initial

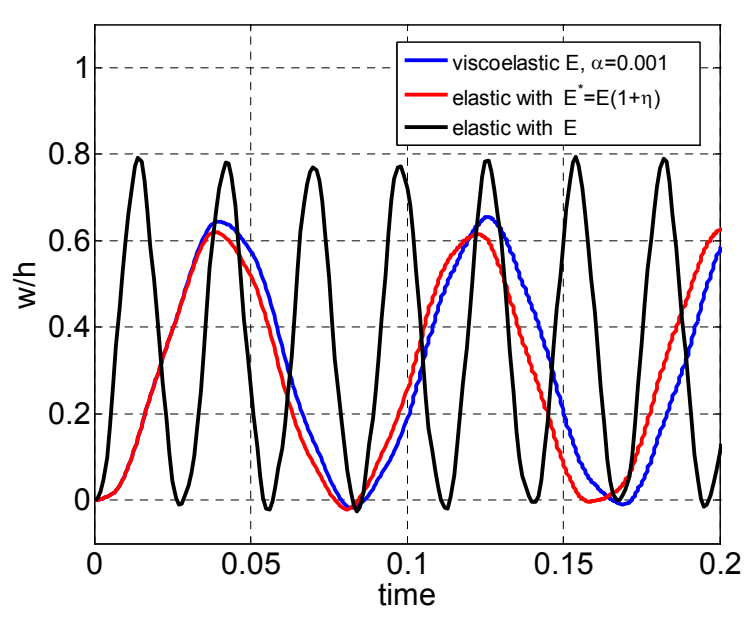

Fig. (9). Time history of the deflection $w(t)$ at the center of the elastic plate with elastic modulus E, $E^{*}=(1+\eta) E$ and viscoelastic plate with $\eta=0.5 \alpha=0.001 ; p_{z}=100 H(t)$.

conditions. Fig. (10) presents the maximum transverse deflection at the center of the plate versus the excitation frequency $\Omega$ for various values of the viscoelastic parameters. Note that for the viscoelastic plate the maximum deflection is taken at the steady state response. It is observed that as the excitation frequency approaches the lower natural frequency of the plate $\left(\omega_{1}=53.42\right)$ the deflection increases and remains bounded due to the viscoelastic behavior of the material. Due to the nonlinear character of the problem two stable periodic solutions are observed for some values of the excitation frequency. Moreover, a jump phenomenon appears as the excitation frequency increases or decreases. The amplitude curves move to the left in case (i) indicating a softening character of the system, while in case (ii) the curves move to the right indicating a hardening nonlinear behavior. Fig. (11) presents the time history of the deflection at the center of the plate for the elastic and the viscoelastic material and two values of the excitation frequency $\left(\Omega_{1}=56.5, \Omega_{2}=55.5\right.$ ) just before and after the jump. The change of the frequency takes place at $t=4 \mathrm{sec}$ and $t=20 \mathrm{sec}$, respectively. Fig. (12) presents the two stable solutions for the viscoelastic plate $(\alpha=1, \eta=0.001)$ for $\Omega=52$ resulting from two different initial conditions. The dependence of the maximum deflection at the steady state response of the viscoelastic plate on the initial central deflection is shown in Fig. (13). The first mode shape is taken as initial deflection.

Example 3. Resonance of a viscoelastic cantilever plate

The cantilever plate of Fig. (14) is subjected to a uniform transverse load $p_{z}=0.0001 k \cos (\Omega t)$ where $k$ is the amplitude parameter and $\Omega$ the excitation frequency. The thickness of the plate is $h=0.01 \mathrm{~m}$ while the parameters of the material are $E=21 \times 10^{6} \mathrm{~N} / \mathrm{m}^{2}, \quad \rho=7550 \mathrm{~kg} / \mathrm{m}^{3}$, $\nu=0.3, \quad \eta=0.03$. The results were obtained using $N=255$ boundary elements, $M=131$ nodes resulting from 205 triangular cells (Fig. 15) and 35 linear modes for 

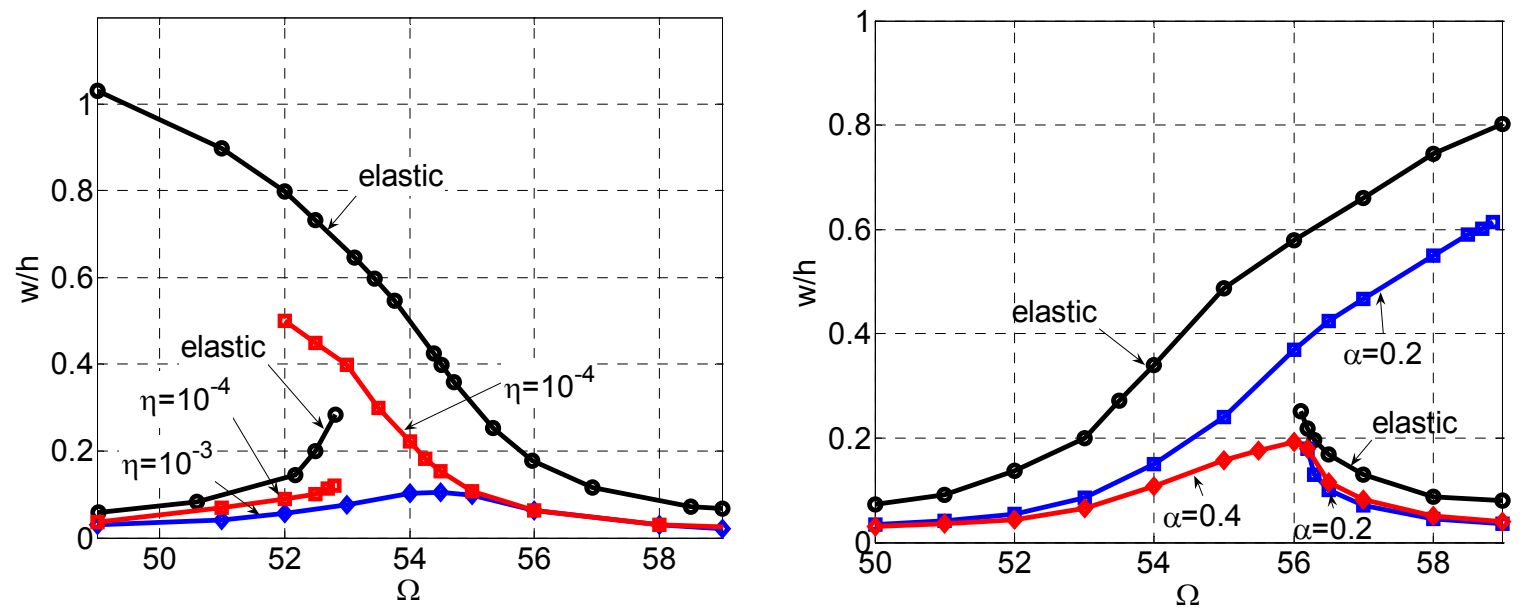

Fig. (10). Amplitude-frequency curves for various values of the viscoelastic parameters and for the two cases of the inplane boundary conditions (i) movable (ii) immovable.
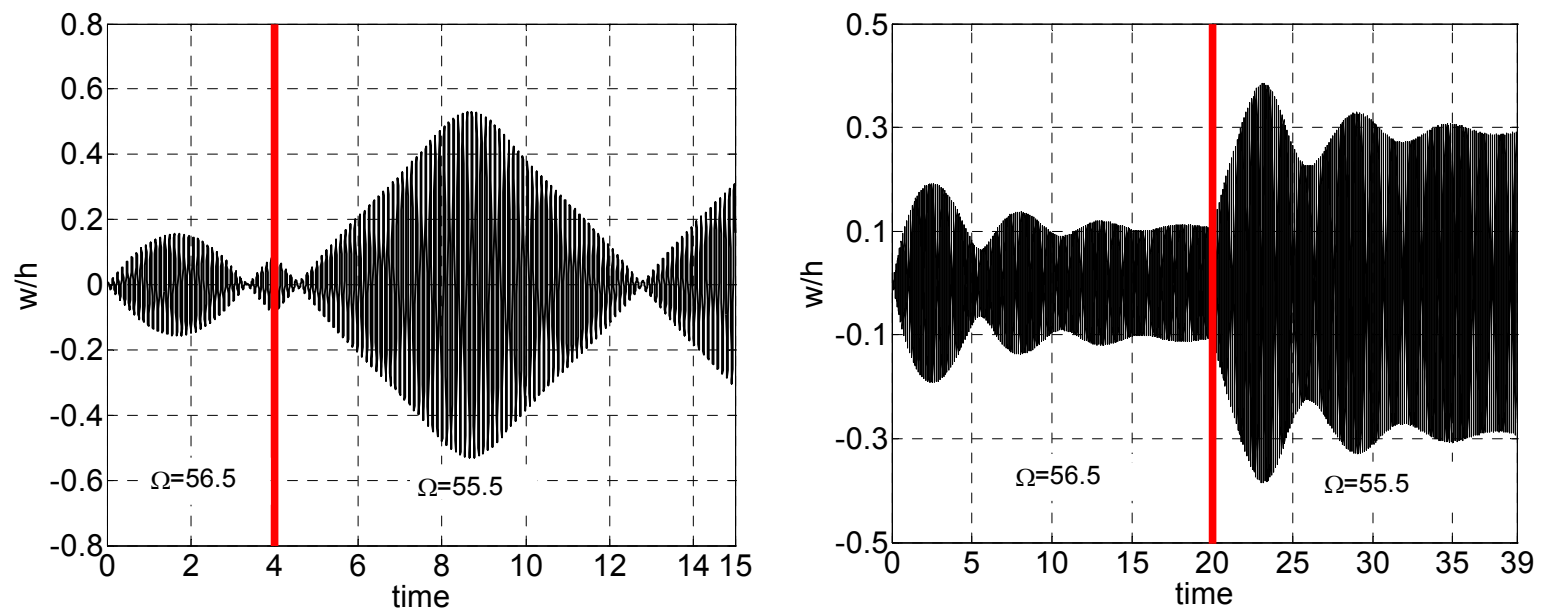

Fig. (11). Time history of deflection at the center of the plate for two values of the excitation frequency $\left(\Omega_{1}=56.5, \Omega_{2}=55.5\right)$ : (a) elastic material, (b) viscoelastic material ( $\alpha=0.2, \eta=0.01$, case (ii)).
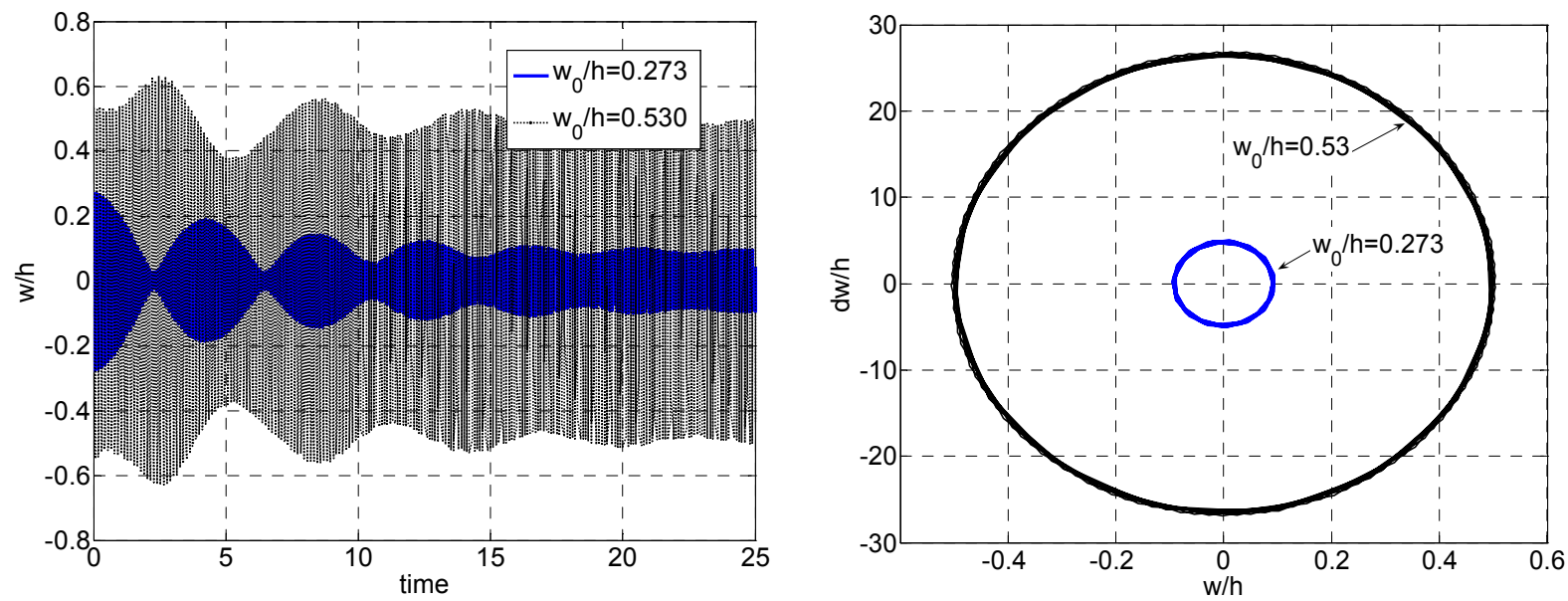

Fig. (12). (a) Time history of the deflection at the center of the plate and (b) phase plane of the steady state response of the two stable solutions $(\alpha=1, \eta=0.001, \omega=52)$. 


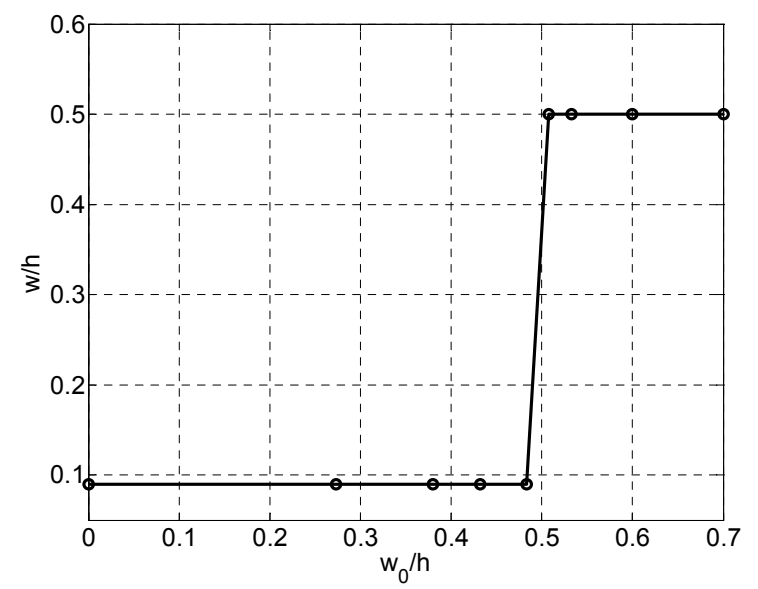

Fig. (13). Influence of initial conditions on the steady state response of the viscoelastic plate in example 2 $(\alpha=1, \eta=0.001, \omega=52$ ).

reduction. Fig. (16) presents the steady state response (transverse deflection at point A) of the plate versus the excitation frequency $\Omega$ for various values of the order of the fractional derivative for constant amplitude parameter $k=1$. The lower natural frequency of the plate is $\omega_{1}=3.12$. It is observed that the amplitude curves move to the right indicating a hardening nonlinear behaviour of the cantilever plate. For $\alpha=0.2$ a jump phenomenon appears. Fig. (17) presents the response of the plate versus the amplitude parameter $k$ for viscoelastic material with $\alpha=0.2$ and for constant frequency excitation $\Omega=3.45$. A hysteretic effect and a jump phenomenon appear for increasing and decreasing amplitude parameter $k$, indicated by the arrows in Fig. (17). The two stable solutions that exist in a certain value of the excitation

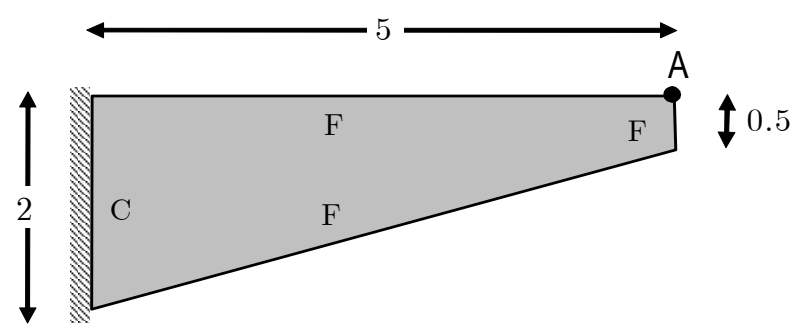

Fig. (14). Boundary conditions of cantilever plate in example3.

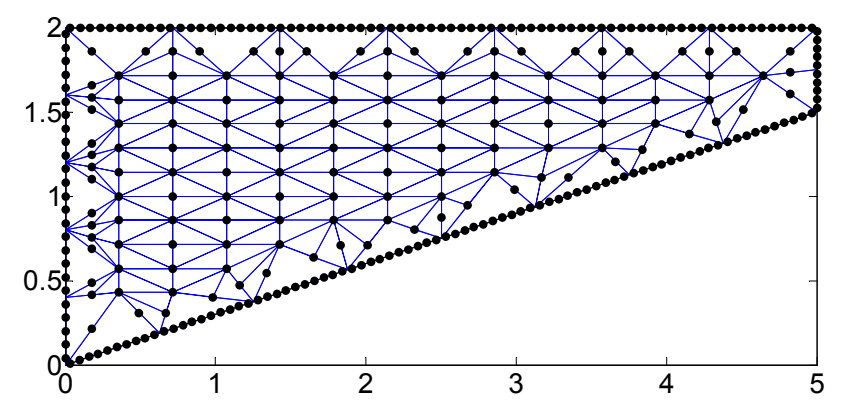

Fig. (15). Boundary and domain discretization of cantilever plate in example 3. frequency (Fig. 16 for $\alpha=0.2$ ) or the amplitude parameter (Fig. 17) are obtained by changing the initial conditions of the plate. Fig. (18) presents the time history of the deflection at point $\mathrm{A}$ for different initial velocities.

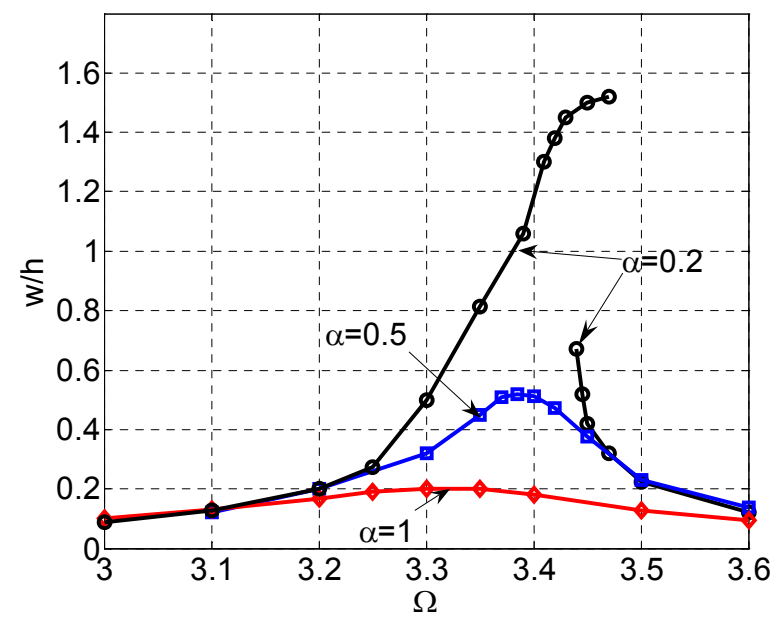

Fig. (16). Amplitude-frequency curves for various values of the order $\alpha$ of the fractional derivative model $(k=1)$.

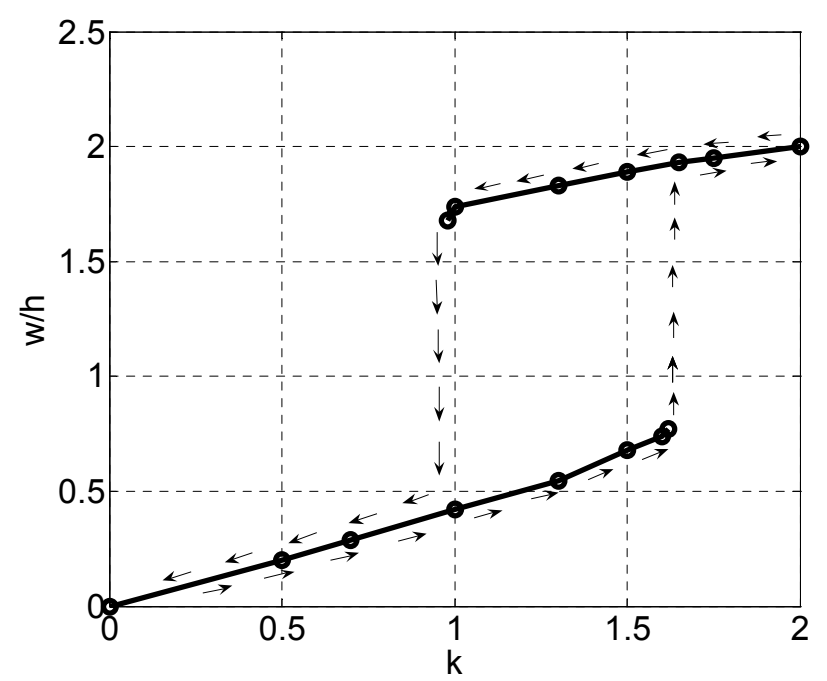

Fig. (17). Amplitude of the response of the plate as a function of the amplitude parameter $k$ of the external excitation $(\Omega=3.45, \alpha=0.2)$.

\section{CONCLUSIONS}

The nonlinear dynamic response of thin viscoelastic plates of fractional derivative model has been investigated. The plate has an arbitrary shape and is subjected to any type of boundary conditions and loading. The membrane inertia forces are also taken into account. The solution is achieved using the AEM, which converts the coupled nonlinear fractional PDEs describing the response of the viscoelastic plate into three uncoupled linear PDEs that can be solved by the BEM. The initial value problem of the resulting semidiscretised nonlinear equations of motion is efficiently solved using (a) the Ritz method for the reduction of the number of the equations and (b) a new time step integration method for fractional differential equations. 


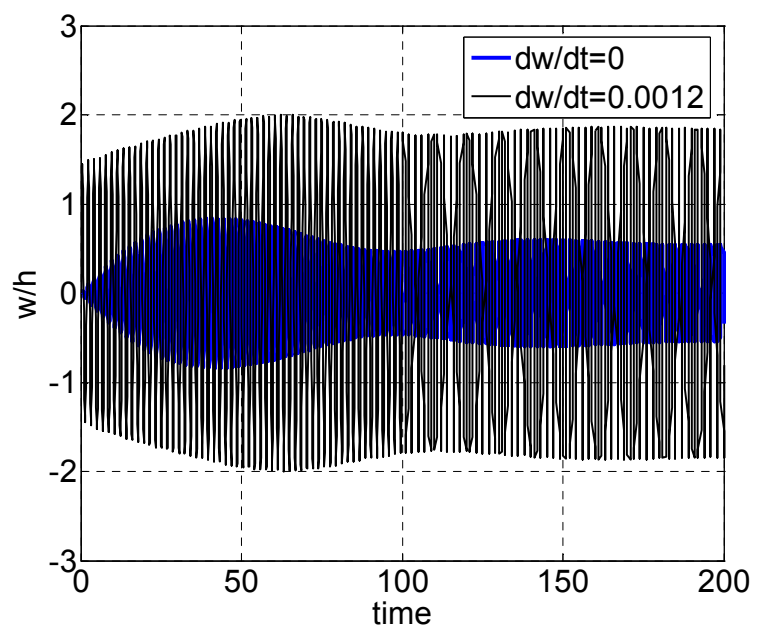

Fig. (18). Time history of the deflection at point A for different initial velocities $(\Omega=3.45, k=1.3, \alpha=0.2)$.

Several plates subjected to various loads and boundary conditions have been analyzed and the influence of the viscoelastic character of the material has been investigated. The nonlinear resonance of thin plates subjected to harmonic excitation is also studied. Due to nonlinear coupling between bending and stretching of the plate a complicated dynamic response occurs similar to that of Duffing-type equation with cubic and quadratic nonlinearities. It was observed that for certain values of the excitation parameters two stable solutions exist which give rise to hysteretic and jump phenomena. The stable periodic solutions are obtained by selecting appropriately the initial conditions of the plate. The viscoelastic character of the material reduces the amplitude of the vibrations at resonance; the amplitude-frequency curves become wider while jump and hysteretic phenomena may disappear. Finally, it was observed that restraining of the inplane displacements along the edges produces a hardening nonlinear response, while a softening response occurs for movable edges. This finding is in accordance with that reported by other investigators.

In closing, the presented solution method offers an efficient computational tool for studying complicated nonlinear dynamic problems of viscoelastic plates. It should be emphasized that the fractional derivative viscoelastic model with $0 \leq \alpha \leq 1$ allows to control the viscoelastic response of the structure; i.e. from pure viscoelastic $(\alpha \rightarrow 1)$ to pure elastic $(\alpha \rightarrow 0)$.

\section{APPENDIX}

\section{Numerical solution of nonlinear FDEs}

The numerical solution of systems of nonlinear fractional differential equations is concisely presented in this Appendix. Detailed description can be found in [23]

We consider the system of the $K$ nonlinear FDEs

$$
\mathbf{F}\left(D_{c}^{\beta} \mathbf{u}, D_{c}^{\alpha} \mathbf{u}, \mathbf{u}\right)=\mathbf{p}(t)
$$

with $0<\alpha<\beta \leq 2, \quad t>0, \mathbf{a}_{i} \in \mathbb{R}, \operatorname{det}\left(\mathbf{a}_{1}\right) \neq 0$

under the initial conditions

$\mathbf{u}(0)=\mathbf{u}_{0}, \quad$ if $\beta \leq 1$

or

$\mathbf{u}(0)=\mathbf{u}_{0}, \dot{\mathbf{u}}(0)=\dot{\mathbf{u}}_{0}$, if $1<\beta \leq 2$

Let $\mathbf{u}=\mathbf{u}(t)$ be the sought solution of Eq. (A1). Then, if the operator $D_{c}^{\beta}$ is applied to $\mathbf{u}$ we have

$$
D_{c}^{\beta} \mathbf{u}=\mathbf{q}(t), \quad 0<\beta \leq 2, \quad t>0
$$

where $\mathbf{q}(t)$ is a vector of fictitious sources, which is unknown in the first instance. Eq. (A3) is the analog equation of (A1). It indicates that the solution of Eq. (A1) can be obtained by solving Eq. (A3) with the initial conditions (A2), if the $\mathbf{q}(t)$ is first established. This is achieved by working as following.

Using the Laplace transform method we obtain the solution of Eq. (A3) as

$$
\mathbf{u}(t)=\mathbf{u}_{0}+[\operatorname{ceil}(\beta)-1] \dot{\mathbf{u}}_{0} t+\frac{1}{\Gamma(\beta)} \int_{0}^{t} \mathbf{q}(\tau)(t-\tau)^{\beta-1} d \tau
$$

where $\operatorname{ceil}()$ represents the ceiling function, e.g. $\operatorname{ceil}(\beta)$ yields the integer greater or equal to $\beta$. The use of this function permits to realize computationally the proper initial conditions prescribed by Eqs (A2). Eq. (A4) is an integral equation for $\mathbf{q}(t)$, which can be solved numerically within a time interval $[0, T]$ as following. The interval $[0, T]$ is divided into $N$ equal intervals $\Delta t=h, h=T / N$, in which $\mathbf{q}(t)$ is assumed to vary according to a certain law, e.g. constant, linear etc. In this analysis $\mathbf{q}(t)$ is assumed to be constant and equal to the mean value in each interval $h$. Hence, Eq. (A4) at instant $t=n h$ can be written as

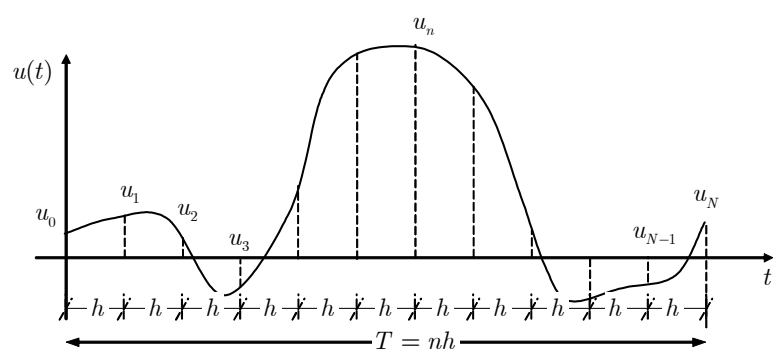

Fig. (A1). Discretization of the interval $[0, T]$ into $N$ equal intervals $h=T / N$.

$$
\begin{aligned}
\mathbf{u}_{n}= & \mathbf{u}_{0}+[\operatorname{ceil}(\beta)-1] n h \dot{\mathbf{u}}_{0}+\frac{1}{\Gamma(\beta)}\left[\mathbf{q}_{1}^{m} \int_{0}^{h}(n h-\tau)^{\beta-1} d \tau\right. \\
& \left.+\mathbf{q}_{2}^{m} \int_{h}^{2 h}(n h-\tau)^{\beta-1} d \tau+\cdots+\mathbf{q}_{n}^{m} \int_{(n-1) h}^{n h}(n h-\tau)^{\beta-1} d \tau\right]
\end{aligned}
$$

which after evaluation of the integrals yields 


$$
\begin{aligned}
\mathbf{u}_{n}= & \mathbf{u}_{0}+[\operatorname{ceil}(\beta)-1] n h \dot{\mathbf{u}}_{0} \\
& +c \sum_{r=1}^{n-1}\left[(n+1-r)^{\beta}-(n-r)^{\beta}\right] \mathbf{q}_{r}^{m}+\frac{c}{2}\left(\mathbf{q}_{n-1}+\mathbf{q}_{n}\right)
\end{aligned}
$$

where

$$
c=\frac{h^{\beta}}{\beta \Gamma(\beta)}, \mathbf{q}_{r}^{m}=\frac{1}{2}\left(\mathbf{q}_{r-1}+\mathbf{q}_{r}\right)
$$

Eq. (A6) can be further written as

$$
\begin{aligned}
-\frac{c}{2} \mathbf{q}_{n}+\mathbf{u}_{n} & =\mathbf{u}_{0}+[\operatorname{ceil}(\beta)-1] n h \dot{\mathbf{u}}_{0} \\
& +c \sum_{r=1}^{n-1}\left[(n+1-r)^{\beta}-(n-r)^{\beta}\right] \mathbf{q}_{r}^{m}+\frac{c}{2} \mathbf{q}_{n-1}
\end{aligned}
$$

We now set

$D_{c}^{\alpha} \mathbf{u}=\overline{\mathbf{q}}(t)$

where $\overline{\mathbf{q}}(t)$ is another unknown vector. We can establish a relation between $\mathbf{q}(t)$ and $\overline{\mathbf{q}}(t)$ by considering the Laplace transform of Eqs (A3) and (A9). Thus, we can write

$$
\begin{aligned}
& \mathbf{U}(s)=\mathbf{u}_{0} \frac{1}{s}+[\operatorname{ceil}(\beta)-1] \dot{\mathbf{u}}_{0} \frac{1}{s^{2}}+\frac{1}{s^{\beta}} \mathbf{Q}(s) \\
& \mathbf{U}(s)=\mathbf{u}_{0} \frac{1}{s}+[\operatorname{ceil}(\alpha)-1] \dot{\mathbf{u}}_{0} \frac{1}{s^{2}}+\frac{1}{s^{\alpha}} \overline{\mathbf{Q}}(s)
\end{aligned}
$$
have

Equating the right-hand sides of the above equations we

$$
\overline{\mathbf{Q}}(s)=([\operatorname{ceil}(\beta)-\operatorname{ceil}(\alpha)]) \dot{\mathbf{u}}_{0} \frac{1}{s^{2-\alpha}}+\frac{1}{s^{\beta-\alpha}} \mathbf{Q}(s), \alpha \neq \beta
$$

Taking the inverse Laplace transform of Eq. (A11) we obtain

$$
\begin{aligned}
& \overline{\mathbf{q}}=([\operatorname{ceil}(\beta)-\operatorname{ceil}(\alpha)]) \dot{\mathbf{u}}_{0} \frac{t^{1-\alpha}}{\Gamma(2-\alpha)}+\frac{1}{\Gamma(\beta-\alpha)} \\
& \int_{0}^{t} \mathbf{q}(\tau)(t-\tau)^{\beta-\alpha-1} d \tau
\end{aligned}
$$

Using the same disctetization of the interval $[0, T]$ to approximate the integral in Eq. (A12), we obtain

$$
\begin{aligned}
\overline{\mathbf{q}}_{n}= & ([\operatorname{ceil}(\beta)-\operatorname{ceil}(\alpha)]) n^{1-\alpha} d \dot{\mathbf{u}}_{0} \\
& +\bar{c} \sum_{r=1}^{n}\left[(n+1-r)^{\beta-\alpha}-(n-r)^{\beta-\alpha}\right] \mathbf{q}_{r}^{m}
\end{aligned}
$$

where

$$
\bar{c}=\frac{h^{\beta-\alpha}}{(\beta-\alpha) \Gamma(\beta-\alpha)}, d=\frac{h^{1-\alpha}}{\Gamma(2-\alpha)}, \mathbf{q}_{r}^{m}=\frac{1}{2}\left(\mathbf{q}_{r-1}+\mathbf{q}_{r}\right)
$$

Eq. (A13) can be further written as

$$
\begin{aligned}
& -\frac{\bar{c}}{2} \overline{\mathbf{q}}_{n}+\mathbf{q}_{n}=[\operatorname{ceil}(\beta)-\operatorname{ceil}(\alpha)] n d \dot{\mathbf{u}}_{0} \\
& \quad+\bar{c} \sum_{r=1}^{n-1}\left[(n+1-r)^{\beta-\alpha}-(n-r)^{\beta-\alpha}\right] \mathbf{q}_{r}^{m}+\frac{\bar{c}}{2} \mathbf{q}_{n-1}
\end{aligned}
$$

Applying Eq. (A1) for $t=t_{n}$ we have

$\mathbf{F}\left(\mathbf{q}_{n}, \overline{\mathbf{q}}_{n}, \mathbf{u}_{n}\right)=\mathbf{p}_{n}$

Eqs (A8), (A15) and (A16) are algebraic equations and they can be combined and solved successively for $n=1,2, \ldots$ to yield the solution $\mathbf{u}_{n}$ and the fractional derivatives $\mathbf{q}_{n}, \overline{\mathbf{q}}_{n}$ at instant $t=n h \leq T$. For $n=1$, the value $\mathbf{q}_{0}$ appears in the right hand side of Eqs (A8) and (A15). This value can be evaluated as following.

Equation (A16) for $t=0$ gives

$\mathbf{F}\left(\mathbf{q}_{0}, \overline{\mathbf{q}}_{0}, \mathbf{u}_{0}\right)=\mathbf{p}_{0}$

The above equation includes two unknowns, $\mathbf{q}_{0}, \overline{\mathbf{q}}_{0}$. These values can be expressed in terms of the known initial conditions using the relations below [23].

$$
\left.\begin{array}{l}
\overline{\mathbf{q}}_{0}=\left[\mathbf{a}_{1} \frac{\Gamma(2-\alpha)\left(2-2^{1-\beta}\right)}{\Gamma(2-\beta)\left(2-2^{1-\alpha}\right)} h^{\alpha-\beta}+\mathbf{a}_{2}\right]^{-1}\left(\mathbf{p}_{0}-\mathbf{a}_{3} \mathbf{u}_{0}\right) \\
\mathbf{q}_{0} \cong \frac{\Gamma(2-\alpha)}{\Gamma(2-\beta)} \frac{\left(2-2^{1-\beta}\right)}{\left(2-2^{1-\alpha}\right)} h^{\alpha-\beta} \overline{\mathbf{q}}_{0} \\
0<\alpha<\beta \leq 1 \\
\overline{\mathbf{q}}_{0}=\left[\mathbf{a}_{1} \frac{\Gamma(3-\alpha)\left(2-2^{2-\beta}\right)}{\Gamma(3-\beta)\left(2-2^{2-\alpha}\right)} h^{\alpha-\beta}+\mathbf{a}_{2}\right]^{-1}\left(\mathbf{p}_{0}-\mathbf{a}_{3} \mathbf{u}_{0}\right) \\
\mathbf{q}_{0} \cong \frac{\Gamma(3-\alpha)}{\Gamma(3-\beta)} \frac{\left(2-2^{2-\beta}\right)}{\left(2-2^{2-\alpha}\right)} h^{\alpha-\beta} \overline{\mathbf{q}}_{0} \\
1<\alpha<\beta \leq 2 \\
\overline{\mathbf{q}}_{0}=\frac{1}{\Gamma(2-a)} h^{1-a}\left(2-2^{1-\alpha}\right) \dot{\mathbf{u}}_{0} \\
\mathbf{q}_{0}=\mathbf{a}_{1}^{-1}\left(\mathbf{p}_{0}-\mathbf{a}_{3} \mathbf{u}_{0}-\mathbf{a}_{2} \overline{\mathbf{q}}_{0}\right) \\
0<\alpha \leq 1 \text { and } 1<\beta \leq 2
\end{array}\right\}
$$

\section{REFERENCES}

[1] Stiassnie M. On the application of fractional calculus for the formulation of viscoelastic models. Appl Math Model 1979; 3: 30002.

[2] Haneczok G, Weller M. A fractional model of viscoelastic relaxation. Mater Sci Eng A 2004; 370: 209-12.

[3] Ramirez LES, Coimbra CFM. A variable order constitutive relation for viscoelasticity. Ann Phys (Leipzig) 2007; 16: 543-52.

[4] Nayfeh AH, Mook DT. Nonlinear oscillations. New York: Wiley 1979.

[5] Fang T, Dowell EH. Numerical simulation of jump phenomena in stable Duffing systems. Int J Non-Linear Mech 1987; 22: 267-74.

[6] Chu H-N, Herrmann G. Influence of large amplitude on free flexural vibrations of rectangular elastic plates. J Appl Mech 1956; 23: 532-40. 
[7] Sridhar S, Mook DT, Nayfeh AH. Non-Linear resonances in the forced responses of plates, Part I: symmetric responses of circular plates. Sound Vib 1975; 41: 359-73.

[8] Amabili M. Nonlinear vibrations of rectangular plates with different boundary conditions: theory and experiments. Comp Struct 2004; 82: 2587-650.

[9] Parzygnat WJ, Pao Y-H. Resonance phenomena in the nonlinear vibration of plates governed by Duffing's equation. Int J Eng Sci 1978; 16: 999-1017.

[10] Haterbouch M, Benamar R. Geometrically nonlinear free vibrations of simply supported isotropic thin circular plates. J Sound Vib 2005; 280: 903-24.

[11] Leung AYT, Mao SG. A symplectic Galerkin method for nonlinear vibration of beams and plates. J Sound Vib 1995; 183: 47591.

[12] Lee Y-Y, Ng CF. Nonlinear response of plates using finite element modal reduction method. Eng Struct 2001; 23: 1104-14.

[13] Ribeiro P, Petyt M. Geometrical non-linear, steady state, forced, periodic vibration of plates, part I: model and convergence studies. J Sound Vib 1999; 226: 955-83.

[14] Ribeiro P, Petyt M. Nonlinear vibration of plates by the hierarchical finite element and continuation methods. Int J Mech Sci 1999; 41: 437-59.

[15] Wang X-X, Qian J, Huang M-K. A boundary integral equation formulation for large amplitude nonlinear vibration of thin elastic plates. Comput Methods Appl Mech Eng 1991; 86: 73-86.

[16] Katsikadelis JT, Nerantzaki MS, Kandilas CB. A BEM approach to non-linear vibrations of plates. In: Moan T, Kounadis AN, Krätzig WB, Schuëller GI, Eds. Structural Dynamics-EURODYN ' 93. Proc. of the 2nd European Conference on Structural Dynamics: EURODYN 93; 1993 June 21-23. Trondheim, Norway, Balkema: Rotterdam 1992; pp. 659-71.

[17] Nerantzaki MS, Katsikadelis JT. Nonlinear dynamic analysis of circular plates with varying thickness. Arch Appl Mech 2007; 77: 381-91.
[18] Ioannides E, Grootenhuis P. A finite element analysis of the harmonic response of damped three-layer plates. J Sound Vib 1979; 67: 203-18.

[19] Eshmatov BKh. Nonlinear vibrations and dynamic stability of viscoelastic orthotropic rectangular plates. J Sound Vib 2007; 300: 709-26.

[20] Khodzhaev1 DA, Eshmatov BKh. Nonlinear vibrations of a viscoelastic plate with concentrated masses. J Appl Mech Tech Phys 2007; 48: 905-14.

[21] Rossikhin YuA, Shitikova MV. Analysis of free non-linear vibrations of a viscoelastic plate under the conditions of different internal resonances. Int J Non-Linear Mech 2006; 41: 313-25.

[22] Katsikadelis JT. The analog boundary integral equation method for nonlinear static and dynamic problems in continuum mechanics. $\mathbf{J}$ Theor Appl Mech 2002; 40: 961-84.

[23] Katsikadelis JT. Numerical solution of multi-term fractional differential equations. ZAMM Zeitschrift für Angewandte Math Mech 2009; 89: 593-608.

[24] Gaul L. The influence of damping on waves and vibrations. Mech Syst Signal Proc 1999; 13: 1-30.

[25] Podlubny I. Fractional differential equations. New York: Academic Press 1999.

[26] Malvern LE. Introduction to the Mechanics of a continuum medium. Engelwood Cliffs, New Jersey: Prentice-Hall 1969.

[27] Katsikadelis JT, Babouskos NG. Nonlinear flutter instability of thin damped plates. A solution by the analog equation method. J Mech Mater Struct 2009; 4: 1395-414.

[28] Katsikadelis JT. Special methods for plate analysis. In: Beskos DE, Ed. Boundary element analysis of plates and shells. New York: Springer-Verlag 1991; pp. 221-311.

[29] Thompson JF, Soni BK, Weatherill NP. Handbook of grid generation. USA: CRC Press 1998

[30] Katsikadelis JT. Boundary elements: theory and applications. Amsterdam-London: Elsevier 2002.

[31] Amabili M. Nonlinear vibrations and stability of shells and plates. USA: Cambridge University Press 2008.

(C) Babouskos and Katsikadelis; Licensee Bentham Open.

This is an open access article licensed under the terms of the Creative Commons Attribution Non-Commercial License (http://creativecommons.org/licenses/by-nc/3.0/) which permits unrestricted, non-commercial use, distribution and reproduction in any medium, provided the work is properly cited. 